\title{
PERCEPÇÃO SOBRE O USO DE PLANTAS MEDICINAIS E IMPACTOS NO CERRADO NA REGIÃO DA CIDADE DE GOIÁS (GO)
}

\author{
Debborah Gonçalves Bezerra ${ }^{1}$ \\ Nariel Aparecida de Arruda ${ }^{2}$ \\ Pedro Paulino Borges ${ }^{3}$ \\ Rafael Batista Ferreira ${ }^{4}$ \\ Patrícia Lima D'Abadia ${ }^{5}$ \\ Carlos de Melo e Silva Neto ${ }^{6}$ \\ Murilo Mendonça Oliveira de Souza ${ }^{7}$
}

Resumo: $\mathrm{O}$ uso de plantas medicinais para fins terapêuticos é uma prática utilizada desde os primórdios da humanidade até os dias atuais. O Cerrado é considerado um hotspot de biodiversidade. De toda a diversidade florística existente no bioma há aproximadamente 220 espécies medicinais que são utilizadas diretamente pelo homem. O objetivo do trabalho foi avaliar a percepção de moradores da Cidade de Goiás sobre o uso de plantas medicinais e os impactos ambientais sobre a vegetação do Cerrado. Foram feitas 40 entrevistas com moradores do Município através da abordagem direta com a utilização de um questionário semiestruturado, as perguntas foram relativas ao uso de plantas medicinais e a percepção dos impactos ambientais sobre a vegetação da região. As espécies mais citadas foram Lippia alba, Stryphnodendron adstringens, Lychnophora spp. e Vernonanthura polyanthes, a parte da planta mais utilizada é a folha, seguida da casca. Grande parte das pessoas identificam o Cerrado pelas plantas, a maioria citou Anacardium spp. e Caryocar brasiliense, $92 \%$ delas afirmaram perceber diminuição das plantas medicinais com o passar dos anos, o desmatamento foi apontado como a principal causa, seguido de extrativismo predatório. O uso de plantas medicinais na região da Cidade de Goiás é uma prática comum e importante para a população, no entanto, os entrevistados relataram que com o passar do tempo a vegetação tem diminuído devido a ação do homem. Desse modo torna-se necessário medidas de conscientização e proteção do Cerrado para a manutenção da biodiversidade e da cultura popular relacionada ao uso das plantas medicinais.

Palavras-chave: Conhecimento Popular; Fitoterápicos; Percepção ambiental.

\footnotetext{
1 Universidade Estadual de Goiás. E-mail: debborah.b@gmail.com

2 Universidade Estadual de Goiás. E-mail: narielarrudabio@gmail.com

3 Universidade Estadual de Goiás. E-mail: borgespep@gmail.com

${ }^{4}$ Universidade Estadual de Goiás. E-mail: rafael_g3bf@hotmail.com

5 Universidade Estadual de Goiás. E-mail: patricialima.bio@gmail.com

${ }^{6}$ Instituto Federal de Goiás e Universidade Estadual de Goiás. E-mail: carlos.neto@ifg.edu.br

7 Universidade Estadual de Goiás. E-mail: murilosouza@hotmail.com
} 
Abstract: The use of medicinal plants for therapeutic purposes is a practice used from the earliest days of humanity until to the present days. The Cerrado is considered a biodiversity hotspot. Of all the floristic diversity existing in the biome there are approximately 220 medicinal species that are used directly by human. The aim of this work was to evaluate the perception of population of the City of Goiás about using medicinal plants and the environmental impacts on the Cerrado vegetation. A total of 40 interviews were conducted with residents of the municipality through a direct approach using a semi-structured questionnaire. The questions were related to the use of medicinal plants and the perception about environmental impacts on the vegetation of the place. The most frequently mentioned species were Lippia alba, Stryphnodendron adstringens, Lychnophora spp. and Vernonanthura polyanthes, the most used part of the plant is the leaf, followed by the bark. People identified the Cerrado by plants, most of them cited Anacardium spp. and Caryocar brasiliense, 92\% said they noticed a decrease in medicinal plants over the years, deforestation was pointed out as the main cause, followed by extractivism predatory. The use of medicinal plants in the municipality of Goiás is a common and important practice for population, however, the interviewees reported that over the time vegetation has decreased due to the action of human being. In this way, it becomes necessary measures of awareness and protection of the Cerrado for the maintenance of biodiversity and popular culture related to the use of medicinal plants.

Keywords: Environmental perception; Herbal medicines; Popular knowledge.

\section{Introdução}

O uso de plantas medicinais surgiu de forma empírica e se confunde com a história do próprio homem. Segundo a Agência Nacional de Vigilância Sanitária (ANVISA), planta medicinal é "espécie vegetal, cultivada ou não, utilizada com propósitos terapêuticos". Apesar do conhecimento popular do uso de plantas medicinais parecer ser um sistema de saúde não oficial, em que participam "raizeiros" e comunidade, ele é fonte de alimentação de estudos etnobotânicos (BRASIL, 2010).

O conhecimento tradicional abrange a utilização de plantas como medicamento, alimento e como componente de rituais e costumes de diferentes populações. Assim, esse conhecimento é aplicado tanto para nutrição em nível domiciliar, como no tratamento de doenças simples ou crônicas graves. Fatores como localização geográfica, tipo da comunidade e suas visões sobre o mundo e a biodiversidade dos ecossistemas, tornam as práticas de saúde tradicional únicas e diversas. Sendo assim, comunidades que se encontram em ecossistemas com características geográficas e disponibilidade de recursos biológicos similares, podem produzir medicamentos naturais semelhantes. No entanto, diferenças na preparação desses medicamentos para manejo de doenças certamente serão encontradas, mediante diferentes conotações socioculturais que envolvem o uso de plantas medicinais (UNNIKRISHNAN; SUNEETHA, 2012).

A utilização terapêutica de plantas medicinais se configura como umas das práticas mais antigas no cuidado da saúde humana (DOS SANTOS et al., 2008). De acordo com a Organização das Nações Unidas para a Alimentação e 
a Agricultura (FAO), em 2050 a população mundial será composta por 9,1 bilhões de habitante e cerca de $80 \%$ dessa população dependerá das plantas medicinais para cuidados primários com a saúde (RAMAKRISHNAN et al., 2017).

Em países em desenvolvimento, segundo a Organização Mundial da Saúde, grande parte da população utiliza a fitoterapia popular para aliviar ou mesmo curar diversas enfermidades. Devido, principalmente, a falta de saneamento básico, desnutrição e dificuldade de acesso a medicamentos industrializados, essa parcela da população busca espécies de plantas disponíveis no ambiente, as quais possuem baixo custo e são de livre acesso (MACÊDO et al., 2016).

Apesar do expressivo avanço da indústria farmacêutica no Brasil, o aumento no uso dessas plantas vem ocorrendo devido a fatores como: alto custo dos produtos farmacêuticos, dificuldade de acesso ao Sistema Único de Saúde (SUS), baixo poder econômico para acesso ao sistema particular de saúde, dificuldade de locomoção da população rural até os centros urbanos, facilidade de acesso às plantas pela população geral, além da tendência natural em se utilizar recursos naturais como uma alternativa aos produtos sintéticos (BADKE et al., 2011; BATTISTI et al., 2013).

Por meio da portaria n.․ 2.761, de 19 de novembro (BRASIL, 2013), instituiu-se a Política Nacional de Educação Popular em Saúde no âmbito do SUS, com o objetivo de haver o envolvimento social da população no processo de educação e saúde. Como método complementar o governo federal aprovou a Política Nacional de Plantas Medicinais e Fitoterápicos (BRASIL, 2006), como parte essencial das políticas públicas de saúde, meio ambiente, desenvolvimento econômico e social.

Visando o amadurecimento do uso de fitoterápicos e farmácias vivas foram lançados o Formulário de Fitoterápicos da Farmacopeia Brasileira (BRASIL, 2011), o Memento de Fitoterápicos da Farmacopeia Brasileira (BRASIL, 2016) e o Formulário de Fitoterápicos Farmacopeia Brasileira - $1^{\text {a }}$ edição - Primeiro Suplemento (BRASIL, 2018a). Estes contêm monografias baseadas em evidências científicas. Entretanto, a maioria das monografias descrevem espécies introduzidas ou cultivadas, estando em menor número os trabalhos acerca de plantas medicinais de origem brasileira.

No Brasil há várias regiões em abundância de espécies medicinais: Floresta Amazônica, Mata Atlântica e Campos sulinos, Pantanal Matogrossense, Cerrado, Caatinga, Zona Costeira e Marinha. Nessas regiões são encontradas plantas indicadas popularmente com interesse medicinal, entretanto para a maioria ainda não foram realizados estudos químicos, farmacológicos ou toxicológicos e nem incluídas em compêndios oficiais (BRASIL, 2002).

O Cerrado é considerado um hotspot de biodiversidade, pois possui elevado endemismo e está sob intensa ameaça. As plantas do bioma 
destacam-se por sua elevada diversidade, estima-se que $40 \%$ das espécies vegetais são endêmicas (MYERS et al., 2000). De toda a diversidade florística existente no Cerrado há aproximadamente 220 espécies medicinais que são utilizadas diretamente pelo homem. Além disso, as plantas têm diversas outras utilidades, uma vez que nos prestam variados serviços ecossistêmicos, tais como: recuperação de solos degradados, atuam como barreiras naturais contra o vento, proteção do solo contra a erosão, controle de pragas, entre outros. Apresenta também importância na geração de renda, pois alguns frutos têm importância econômica e social, como por exemplo: Pequi (Caryocar brasiliense Cambess.), Buriti (Mauritia flexuosa L. f.), Mangaba (Hancornia speciosa Gomes), Cagaita (Eugenia dysenterica DC.), Bacupari (Salacia crassifolia (Mart. ex Schult.) G. Don), Cajuzinho do cerrado (Anacardium ssp.), Araticum (Annona crassifolia Mart.) e as sementes do Barú (Dipteryx alata Vogel) (BRASIL, 2018b).

As plantas do Cerrado possuem um alto valor terapêutico para grande parte da população, em especial para pessoas que residem em regiões distantes das áreas urbanas (TOLEDO et al., 2011). Nos últimos anos, a exploração da flora do Cerrado tem crescido significativamente, principalmente devido a novas práticas realizadas por associações comunitárias, as quais utilizam extratos ou partes das plantas para produção de medicamentos. Dentre esses produtos terapêuticos, destacam-se: as pomadas, os xaropes, as soluções tópicas cicatrizantes e antifúngicos, os vermífugos, dentre outros (SOUZA; FELFILI, 2006). A ampla utilização de espécies do Cerrado na medicina popular se dá devido suas características morfológicas, como suas cascas e xilopódios, que retém, acumulam água e nutrientes, e frequentemente, se destacam por possuírem substâncias farmacologicamente ativas (CONCEIÇÃO et al., 2011).

A megabiodiversidade do Cerrado fornece raízes, cascas, resinas, óleos, folhas, argilas, água e diversos outros produtos naturais, os quais são utilizados pelas populações na medicina popular, para tratamento de diversos males. A utilização desses recursos é norteada pelo conhecimento acumulado e adquirido por raizeiros, mateiros e demais pessoas que adquiriram esse conhecimento tradicional dos antepassados (DIAS; LAUREANO, 2010). Entretanto, essa rica biodiversidade, que contribui com a manutenção da saúde humana e de todo o ecossistema, tem sido perdida, em detrimento da ocupação de suas áreas nativas e uso de seus recursos naturais de forma desordenada e predatória. Portanto, práticas de uso sustentável desses recursos são extremamente necessárias para que se assegure a preservação de recursos genéticos do Cerrado de valor incomparável (PIRES; SANTOS, 2000; PEREIRA et al., 2012).

Assim para garantir à população brasileira 0 acesso seguro e 0 uso racional de plantas medicinais e fitoterápicos, promovendo o uso sustentável da biodiversidade é necessário o reconhecimento de práticas populares que utilizam plantas medicinais e remédios caseiros. Na cidade de Goiás, dado o seu longo histórico de ocupação, a população tem tido contato direto com o 
Cerrado, tal relação é citada no plano diretor da cidade, no qual coloca a expressão homem-Cerrado e apresenta passagens sobre a preservação dos recursos naturais (DELGADO, 2005). Ainda hoje, a população tem uma forte relação com o Cerrado, usufruindo dos serviços que ele oferece, como por exemplo, o uso de plantas para alimentação e fins medicinais. Desse modo, o objetivo geral deste estudo foi avaliar a percepção de moradores do Município de Goiás sobre o uso de plantas medicinais. Além disso, i) traçar um perfil socioeconômico dos entrevistados; ii) determinar o conhecimento dos entrevistados sobre o Cerrado; iii) avaliar a percepção dos moradores sobre as mudanças ambientais ocorridas na vegetação da região ao longo do tempo e iv) caracterizar a importância do Cerrado.

\section{Metodologia}

A coleta de dados foi realizada por meio de entrevistas com a população da Cidade de Goiás, no mês de agosto de 2018. O município que era a antiga capital do Estado se localiza na região leste de Goiás e pertence ao bioma Cerrado. De acordo com o último censo, possui uma estimativa populacional de 24.727 habitantes e uma densidade populacional de 7,96 hab/ $\mathrm{km}^{2}$, além disso, possui um IDH de 0.709 e um PIB per capita de $R \$ 15.998,30$ (IBGE, 2018).

Primeira capital do Estado, ela se originou da exploração do ouro no interior do país empreendida pelos bandeirantes que colonizam o Centro-Oeste (XVIII - XIX) (SILVA; SÁ; SÁ, 2015). A exploração do ouro levou a de ocupação de Goiás em meados de 1726, as margens do Rio Vermelho, o arraial de Sant' Ana. Posteriormente, foi conhecida como Vila Boa de Goyas e mais tarde, Cidade de Goiás (SILVA, 2017). Devido aos seus aspectos históricos, em 2001 tornou-se Patrimônio Cultural da Humanidade, o que fez com que o turismo na região aumentasse, tornando-se um importante motor da economia na cidade (DELGADO, 2005; TEIXEIRA et al, 2012). Nessa região têm a sub-bacia hidrográfica do Rio Vermelho. Este está inserido na região hidrográfica do Tocantins Araguaia, na Bacia Hidrográfica do Rio Araguaia, na porção Centro-Oeste do Estado. Estão inseridas nela a microbacia do corrégo Bacalhau encontram se a sudeste da cidade de Goiás/GO, inserida na subbacia do Rio Vermelho (ROZA DOS SANTOS; VESPUCCI; BAYER, 2017).

A pesquisa foi delineada para a obtenção de informações de pessoas provenientes de diferentes gêneros e classes sociais. Desse modo, foram entrevistados homens e mulheres moradores do centro da cidade, bairros da periferia e zona rural, a qual foi representada pelos agricultores familiares de propriedades particulares e de assentamentos que estavam na Cidade comercializando produtos alimentícios, através das feiras. As entrevistas foram realizadas com 40 pessoas, 21 do sexo masculino e 19 do sexo feminino por meio da abordagem direta e com a utilização de um questionário semiestruturado servindo de roteiro para as entrevistas. As perguntas foram feitas de forma não tendenciosa pelos entrevistadores, e as respostas foram transcritas da mesma forma como eram ditas pela população. 
De modo geral, o objetivo do questionário foi: (1) traçar um perfil socioeconômico dos entrevistados; (2) avaliar o seu conhecimento sobre plantas medicinais do Cerrado; e (3) avaliar a percepção sobre o que é Cerrado. Para tanto, em linhas gerais, foram obtidas as seguintes informações dos entrevistados: setor onde mora, idade, sexo, grau de escolaridade e ocupação; percepção sobre as características da vegetação do Cerrado; utilização de plantas medicinais (nome popular, parte utilizada, indicações, efeitos e aquisição do conhecimento); quem indicou a utilização das plantas; se usa em conjunto com outros medicamentos; se é importante ter comprovação científica; como se dá a aquisição das plantas; percepção sobre a diminuição das plantas medicinais no Cerrado; o motivo da diminuição e a importância do Cerrado para a saúde das pessoas. Após as entrevistas, os dados foram tabulados para análise descritiva (porcentagem) e confecção de figuras.

\section{Resultados e Discussão}

Foram contabilizados 40 entrevistados, sendo $53 \%$ mulheres e $47 \%$ homens. A idade dos entrevistados variou entre 18 até mais de 70 anos, sendo a maioria de 51 a 60 anos ( $n=12 ; 30 \%)$, conforme apresentado no Gráfico 1.

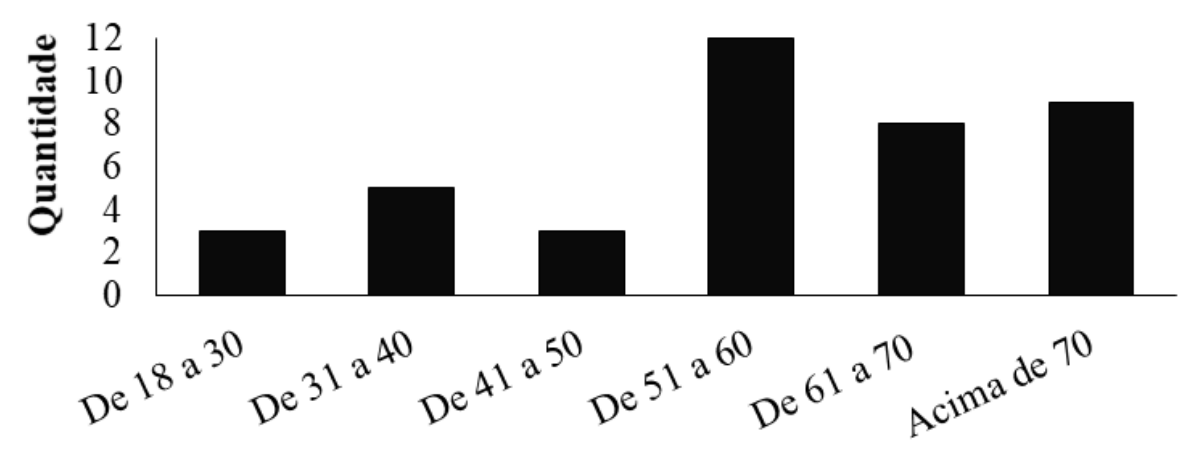

Classes de idade

Gráfico 1: Idade dos entrevistados considerando as classes entre 18 a 30 anos, 31 a 40 anos, 41 a 50 anos, 51 a 60 anos, 61 a 70 anos e acima de 70 anos.

Os entrevistados residem em bairros como Setor Aeroporto $(n=14)$, Centro $(n=10)$, Rio Vermelho $(n=4)$, Santa Bárbara $(n=3)$, João Francisco $(n=$ $2)$, e Bacalhau $(n=1)$. Ademais, 3 entrevistados afirmaram residir em assentamentos próximos à Cidade de Goiás, enquanto 3 residem na Zona Rural do município. Deve-se levar em consideração que a maior parte dos entrevistados já residiram tanto em zona rural quanto na urbana, conforme pode ser visto na Gráfico 2. 


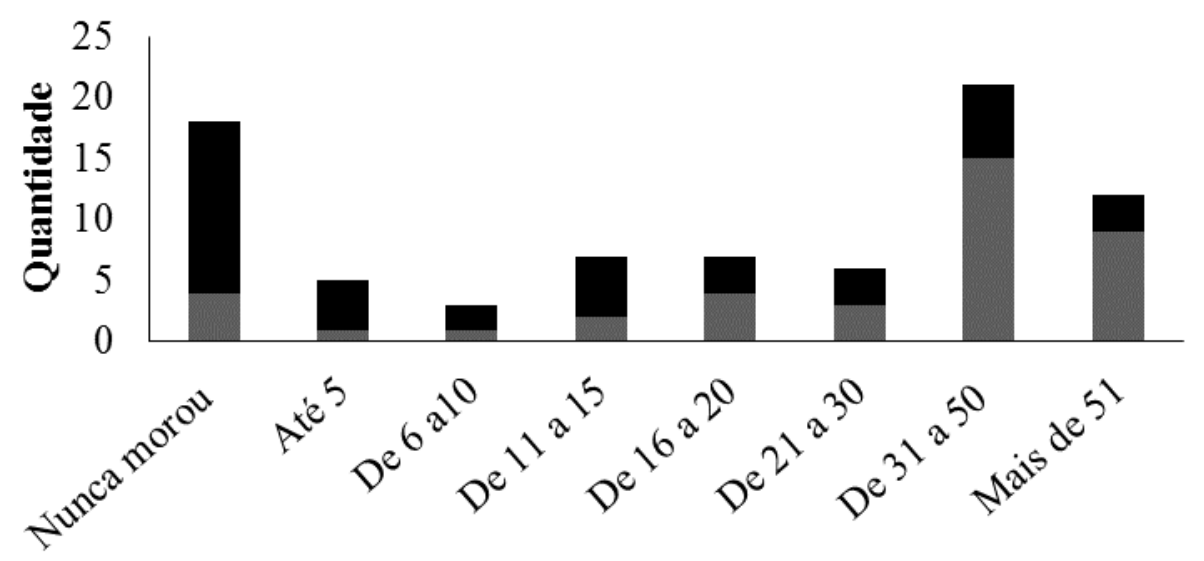

Tempo de moradia na região (anos)

\section{- Urbano Rural}

Gráfico 2: Tempo em que o entrevistado residiu na Zona Rural e na Urbana.

A maior parte dos entrevistados possuem escolaridade entre $01^{\circ}$ ao $5^{\circ}$ ano $(30 \%)$. Entretanto, também foram constatadas as escolaridades entre a $6^{\circ}$ ao $9^{\circ}$ ano (15\%), Ensino Médio completo (15\%), Ensino Superior incompleto $(12,5 \%)$, Ensino Superior completo (10\%), Especialista (5\%) e Doutorado $(2,5 \%)$. Constatou-se que, 4 entrevistados (10\%) não possuem escolaridade. Quanto à profissão dos entrevistados, observa-se pelo Gráfico 3 que a maioria (25\%) trata-se de aposentados.

Grande parte dos entrevistados $(n=37 ; 92,5 \%)$ utilizam plantas com propósitos medicinais, sendo contabilizadas 140 diferentes espécies vegetais utilizadas. Na maioria dos casos o uso dessas plantas está vinculado à indicação de familiares e/ou amigos (85\%). Automedicação e indicações médicas foram citadas em $10 \%$ e $5 \%$ dos casos, respectivamente. A utilização de plantas medicinais, no contexto do grupo familiar abriga um conhecimento próprio, repassado entre as gerações familiares, com particularidades que ficam restritas aquele grupo (CEOLIN et al., 2011). Pinto et al. (2006), ressaltam que a origem do conhecimento das comunidades rurais em relação à utilização de plantas medicinais está ligada às pessoas mais idosas, comungando com a ideia de Ceolin et al. (2011, p.50), que "é entre os membros da família que se propagam informações oralmente quanto a hábitos e cuidados com a saúde, como o uso das plantas medicinais". Percebeu-se que a maioria dos idosos, têm o amplo conhecimento sobre plantas medicinais, devido sua profissão como vendedores de matéria - prima ou formulações com finalidades terapêuticas e alimentícias. 


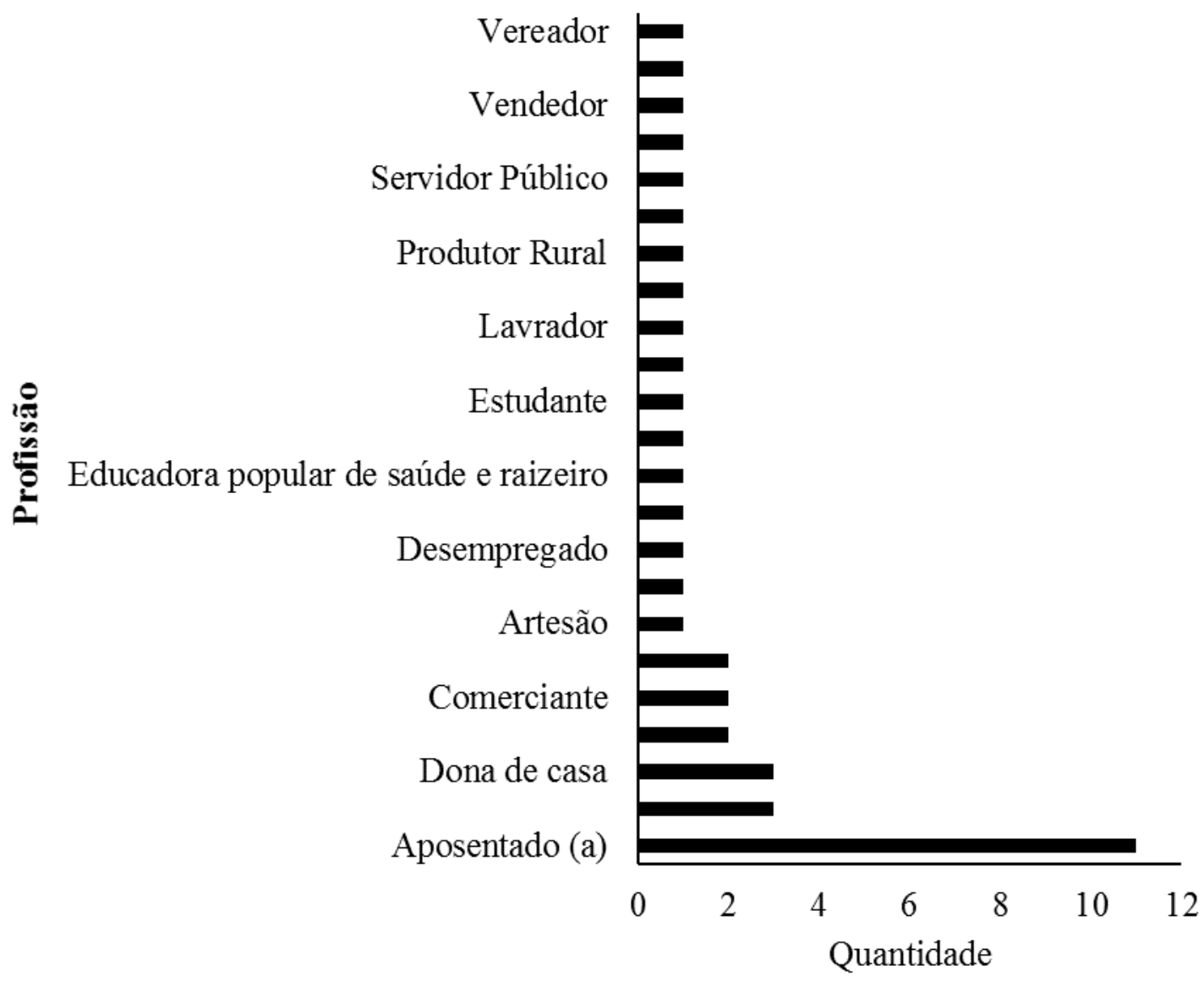

Gráfico 3. Profissão dos entrevistados.

No tocante à relação do uso do conhecimento científico junto com o empírico, como influenciadores da tomada de decisão do uso das plantas medicinais, foi identificado que, poucos entrevistados (5\%) afirmaram que para o uso de plantas medicinais tem-se a necessidade da comprovação científica do benefício causado por essas espécies. Contudo, o uso de plantas medicinais não exime a utilização de outros alopáticos, porquanto $60 \%$ dos entrevistados os associam com medicamentos químicos, conforme apresentado no Gráfico 4. 


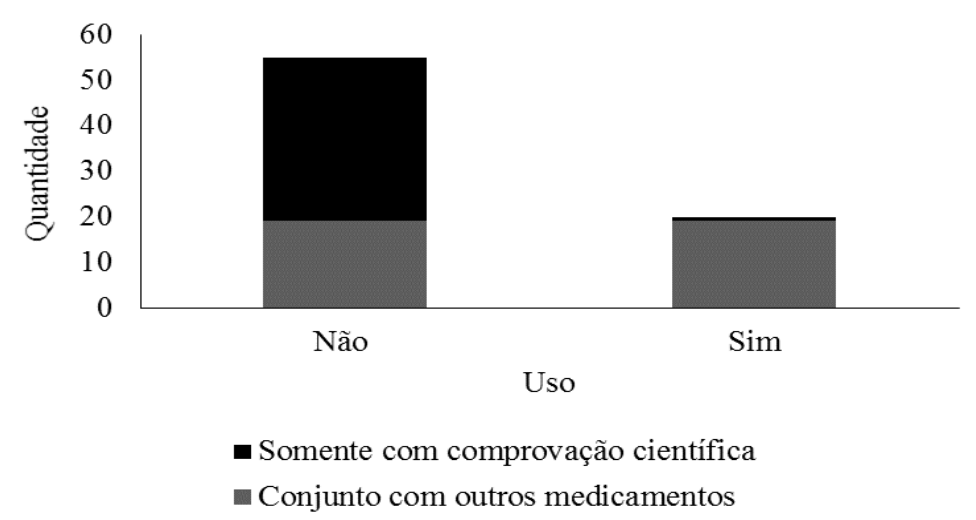

Gráfico 4: Uso das plantas em conjunto com outros medicamentos e importância do conhecimento científico como influenciador do uso das plantas medicinais.

A utilização de plantas medicinais como terapia menos onerosas para o tratamento de enfermidades representa ganho importante nos investimentos humanos e financeiros empregados na área de saúde. Contudo, vários pesquisadores adeptos a utilização de plantas medicinais afirmam que são necessários maiores investimentos em estudos científicos, afim de se levar à eficácia e segurança do uso e na aplicação por parte dos profissionais de saúde, evitando efeitos toxicológicos e associando estrutura-atividade biológica (SANTOS et al., 2012). A pesquisa voltada para o campo das plantas medicinais é eficiente para comprovar suas ações mediante o uso empírico. Deste modo, Argenta et al. (2011, p. 58) asseguram que a pesquisa científica sobre a eficácia das plantas medicinais "torna-se fonte para a relação entre universidade e comunidade seja estabelecida no intuito de melhorar a qualidade de vida da população de baixa renda, principalmente através de uma maior acessibilidade dos recursos terapêuticos disponíveis". A integração destas áreas na pesquisa de plantas medicinais conduz a um caminho promissor e eficaz para descobertas de novos medicamentos.

As plantas medicinais mais citadas estão listadas no Gráfico 5. Observase que, espécies vegetais endêmicas do Cerrado, tais como barbatimão (Stryphnodendron adstringens (Mart.) Coville), arnica (Lychnophora spp. Mart.), assa peixe branco (Vernonanthura polyanthes (Sprengel) Vega \& Dematteis), entre outras, possuem elevada importância medicinal para a população local (Tabela 1 - Material Suplementar). Dentre as plantas citadas na pesquisa, 58 plantas são nativas, 8 são naturalizadas e 26 são introduzidas. Sendo que três espécies nativas pertencem somente ao bioma Cerrado: Pacari (Lafoensia pacari A.St.-Hil.), Carobinha do Campo (Jacaranda decurrens Cham.) e Nó de cachorro (Heteropterys tomentosa A. Juss). Segundo Rodrigues e Carvalho (2001, p. 106), vários são os fatores que influenciam na variedade de espécies utilizadas como plantas medicinais, podendo citar "o preço elevado de medicamentos; anseio no bem-estar e na cura mais rápida, fazendo uso dos dois tipos de tratamentos (medicamentos e remédios naturais); e as contraindicações devido ao uso constante dos medicamentos sintéticos". 


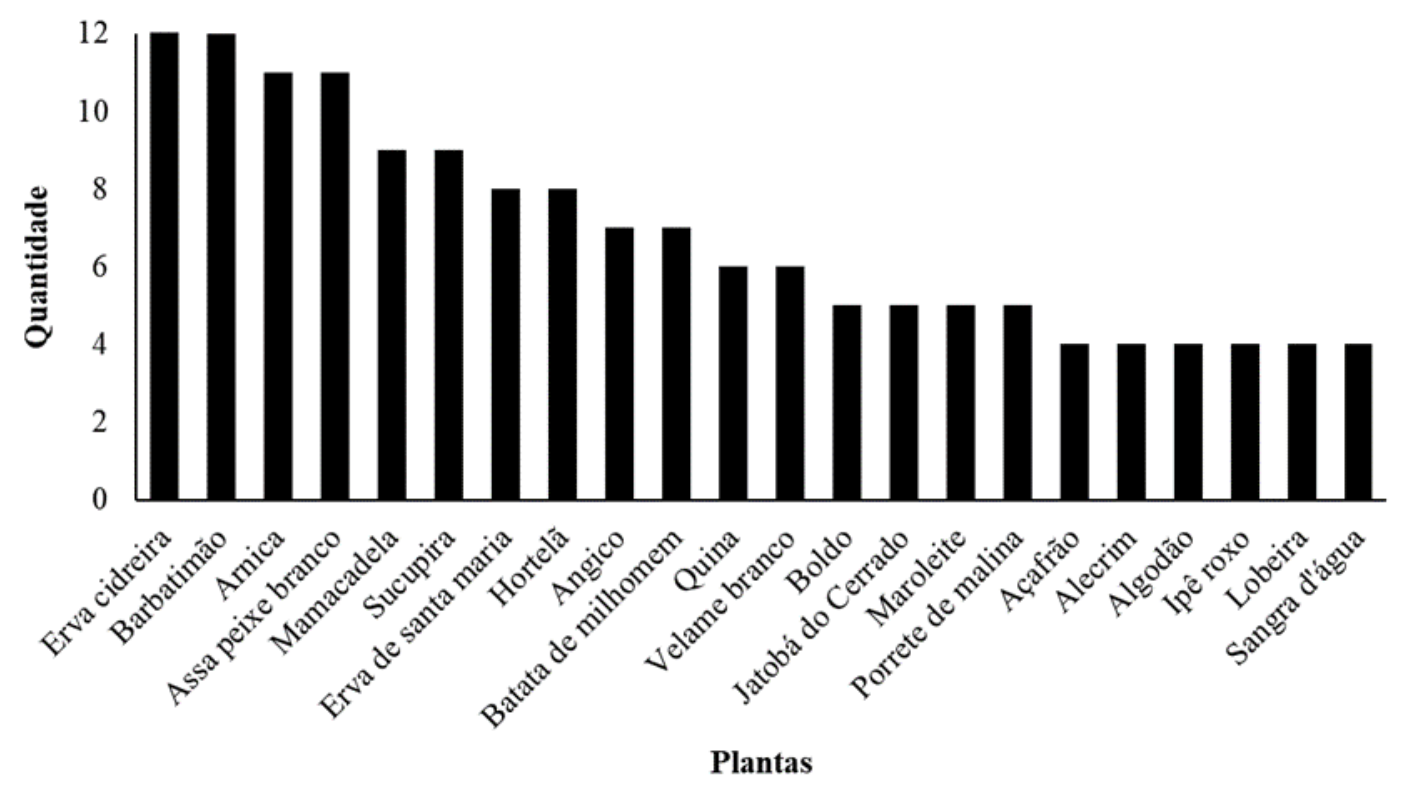

Gráfico 5: Principais plantas (nomes populares) que os entrevistadas utilizam para fins medicinais.

Quanto à parte da planta utilizada, observou-se que as folhas possuem elevada importância (52\%), ademais outras partes das plantas também foram citadas, tais como: casca $(12,5 \%)$, raiz $(10 \%)$, entrecasca $(8,5 \%)$, fruto $(8,5 \%)$ e semente $(8,5 \%)$. É natural que a importância do uso de folhas seja maior do que as outras partes das plantas por que há disponibilidade contínua de folhas; é de fácil acesso e não causa a mortalidade da espécie. Ressalta -se que a utilização de partes das plantas como sementes e frutos, podem auxiliar na preservação dessas espécies, enquanto tende a ocorrer o contrário quando o uso é de parte da planta na qual a extração pode ocasionar a mortalidade da mesma, como por exemplo casca e raiz (ALMEIDA; ALBUQUERQUE, 2002). Em pesquisa realizada por Williams et al. (2000), na qual foi avaliada a comercialização de plantas endêmicas da região de Witwatersrand localizada na África do Sul, foi constatado que, devido a coleta de suas raízes, existe possibilidade de redução da distribuição geográfica de 2/3 das 151 espécies comercializadas no local.

Foram listadas 110 indicações de uso das plantas medicinais, dentre os quais a maior quantidade está relacionada com problemas estomacais (6\%). $\mathrm{O}$ gráfico 6 apresenta as indicações mais citadas pelos entrevistados. As espécies de plantas medicinais mais procuradas são aquelas relacionadas à cura de enfermidades que necessitam de doses diárias de medicamentos, como afirmam Rodrigues e Carvalho (2001). Ademais, observou-se que 94\% das plantas não possuem contraindicações, segundo os entrevistados. A contraindicação mais citada foi o aumento da frequência cardíaca (2\%), com a ressalva do uso em excesso. Outras contraindicações foram: toxicidade $(1 \%)$, aborto $(0,7 \%)$, hemorragia $(0,6 \%)$, aumento da frequência cardíaca $(0,6 \%)$ e não indicado para crianças $(0,6 \%)$. Tomazzoni et al. (2006), afirmam que 
"assim, como as plantas são remédios poderosos e eficazes, o risco de intoxicação causada pelo seu uso indevido deve ser sempre levado em consideração" (p.120). Principalmente, porque muitos têm a ideia de que por ser de fonte natural não faz mal. Assim, há necessidade de conscientização populacional do equilíbrio e uso racional das plantas medicinais.

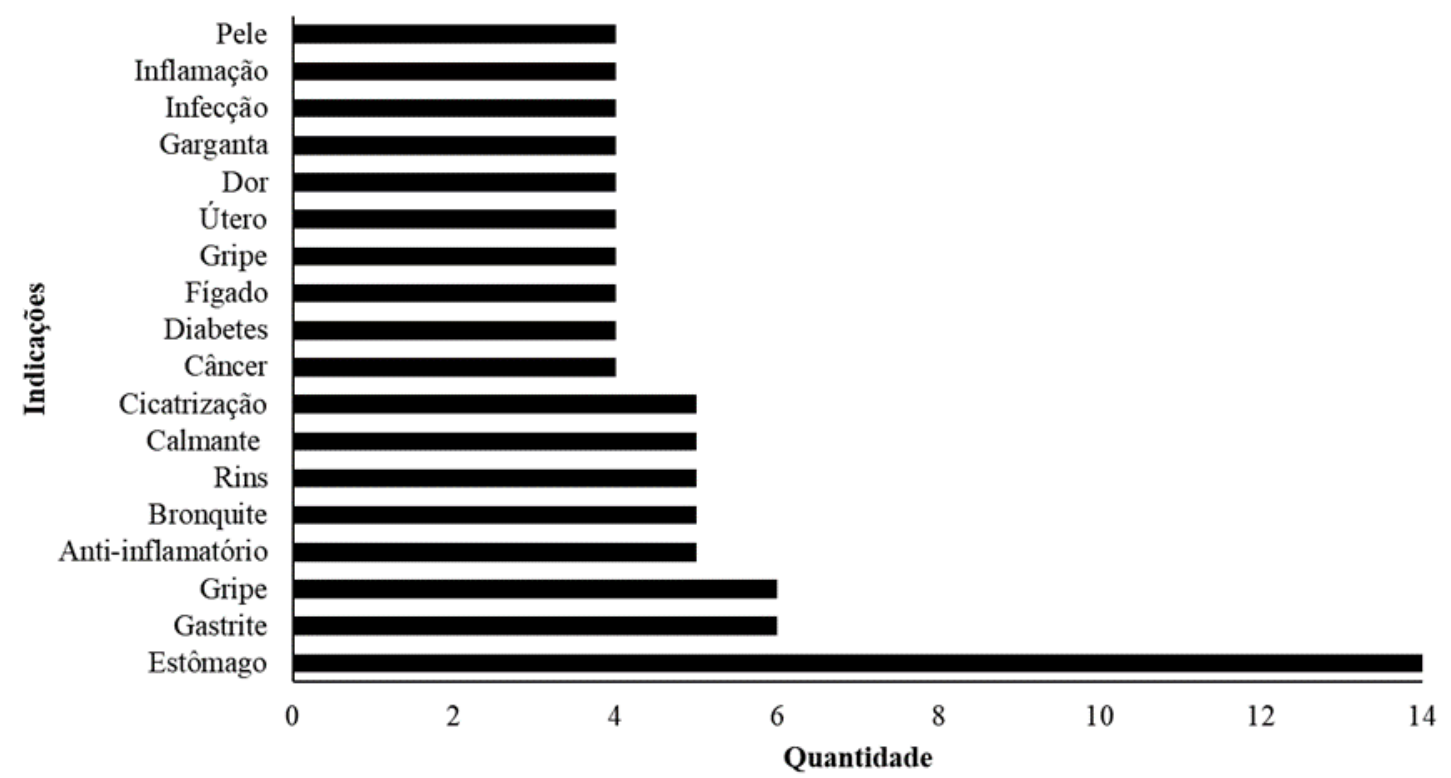

Gráfico 6. Principais indicações que foram citadas das plantas medicinais.

Em relação à percepção ambiental sobre o estado atual do bioma, questionou-se aos entrevistados sobre "as características que identificam o Cerrado". Foi observado que a maioria dos entrevistados relacionou o bioma com as plantas presentes no mesmo, tais como caju, pequi, entre outros, conforme descrito no Gráfico 7, evidenciando a importância socioeconômica das mesmas para a região. Alguns atributos morfológicos das plantas também foram citados, como é o caso de árvores tortas e pequenas. O uso de espécies vegetais oriundas do Cerrado, tanto para uso próprio quanto para comercial, é cotidiano para os moradores da região. Dessa forma, os entrevistados citaram tanto a coleta, a compra e a venda como formas de aquisição dessas plantas. $\mathrm{O}$ fato de existir a possibilidade de coletar in natura plantas endêmicas do Cerrado evidencia a proximidade dos moradores da região com o bioma. 


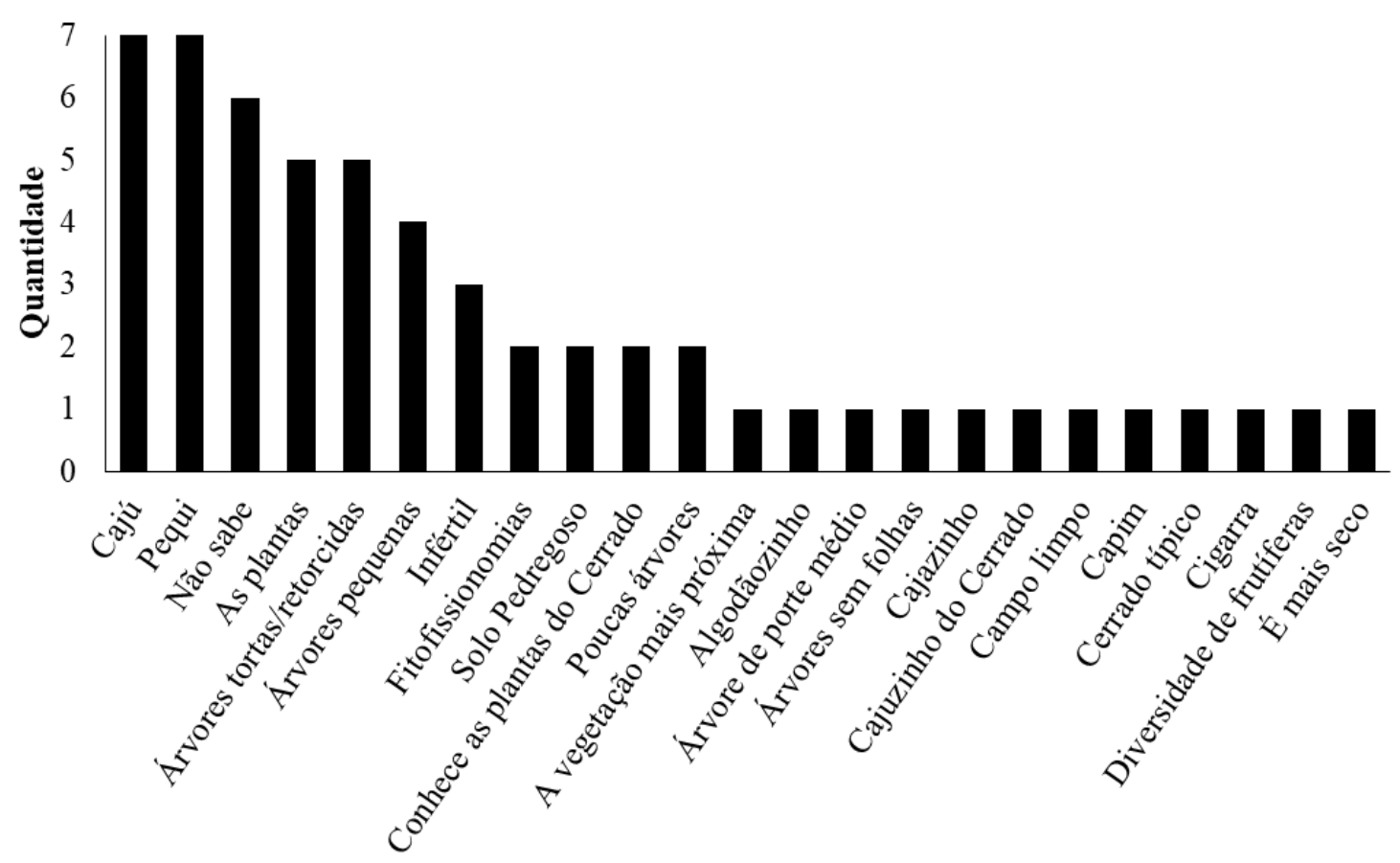

Característica que identifica o Cerrado

Gráfico 7: Características que identificam o bioma Cerrado citadas pelos entrevistados.

Entretanto, deve se levar em consideração que a maior parte dos entrevistados (92\%), afirmam perceber a redução da disponibilidade das plantas endêmicas do Cerrado nos últimos anos. Quando questionado os motivos para que isso ocorra, o desmatamento foi o mais citado (27\%). Outros motivos também foram citados, tais como: extrativismo predatório $(25 \%)$, avanço da agropecuária (17\%), clima (15,5\%) e queimada (15,5\%). Alguns reconheceram que algumas espécies quase já não são encontradas e não há cultivo destas, podendo ser extintas. As respostas obtidas mostram uma percepção ambiental adequada com a realidade da redução do Cerrado nas últimas décadas, uma vez que, mais da metade do bioma já foi perdido principalmente devido aos avanços da agropecuária (KLINK; MACHADO, 2005).

Dentre os fatores responsáveis pela diminuição da disponibilidade de plantas do Cerrado, que foram citados pelos entrevistados, destaca-se o extrativismo predatório de determinadas plantas. Espécies que possuem ação terapêutica eficaz e com alta utilidade, são amplamente exploradas e têm sua disponibilidade no ecossistema rapidamente reduzida (UNNIKRISHNAN; FADEEVA, 2013). Essa tendência de esgotamento de determinados recursos naturais são mais frequentes em países em desenvolvimento, os quais possuem sistemas de saúde precários (UNNIKRISHNAN; SUNEETHA, 2012). Portanto, torna-se urgente a necessidade de desenvolvimento de práticas sustentáveis no uso dessas espécies e a consequente valoração desses recursos. 
Os entrevistados percebem a importância do Cerrado para a região, sendo tal importância relacionada principalmente com aspectos ligados ao consumo de plantas, como é o caso do uso medicinal e alimentício, conforme pode ser visto no Gráfico 8. Deve-se levar em consideração que alguns aspectos relacionados à qualidade do meio ambiente também foram citados, como é o caso de água, ar puro, clima melhor, entre outros.

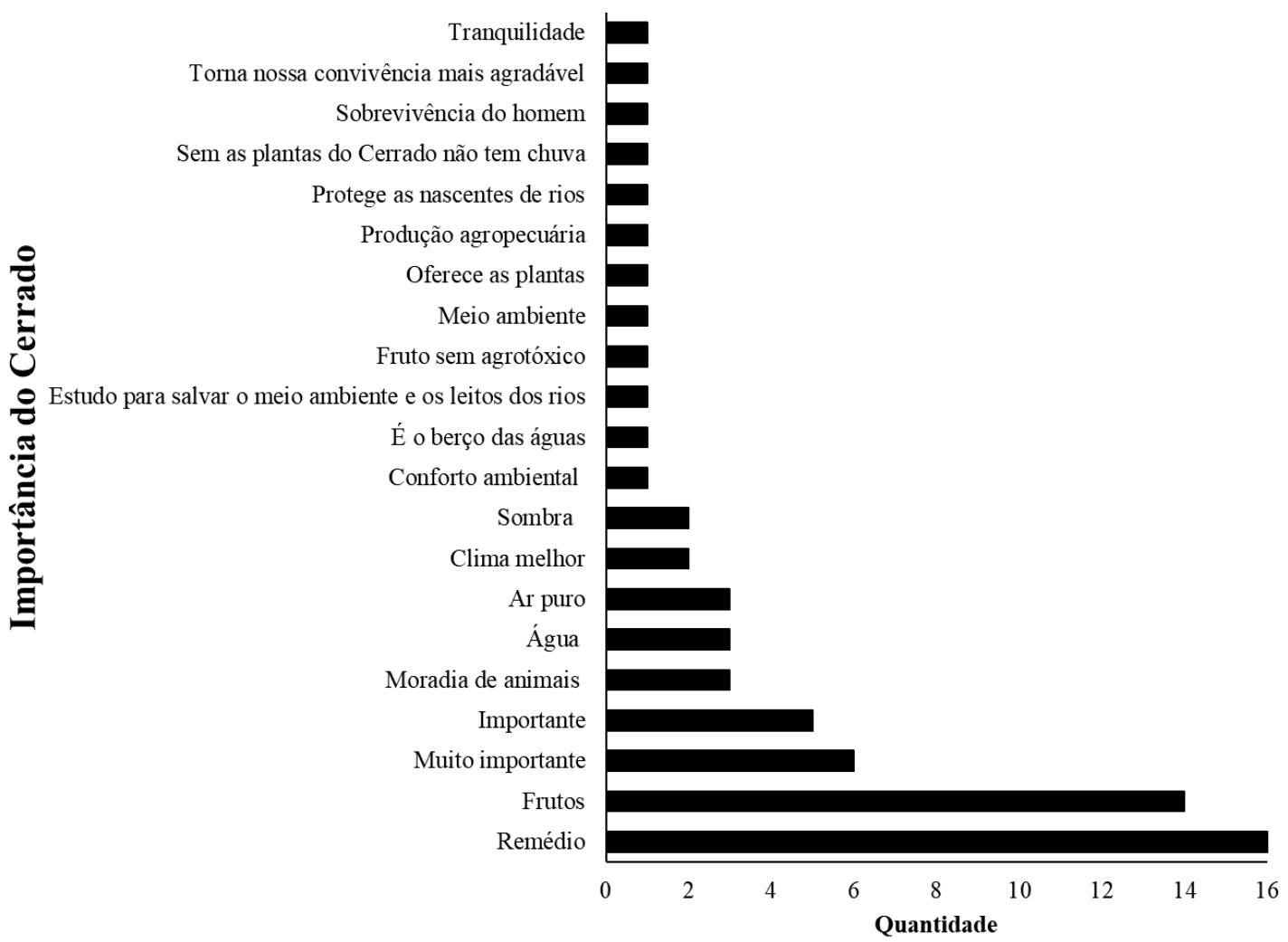

Gráfico 8: Importância do Cerrado de acordo com os relatos obtidos dos entrevistados.

Como a maioria respondeu que faz uso das plantas para tratar enfermidades, justifica-se o fato de grande parte dos entrevistados associarem a importância do Cerrado com remédio. Além disso, o fato de estar na época de frutificação de algumas plantas, pode ter feito com que os entrevistados tenham citado os frutos. Entretanto, outros serviços ambientais menos perceptíveis também foram mencionados.

Os ambientes naturais, como por exemplo o Cerrado, quando se encontram em bom estado de conservação prestam diversos serviços ecossistêmicos, que são essenciais para a sobrevivência do homem, tais como: regulação climática, controle da qualidade do ar, água de boa qualidade e proteção contra propagação de doenças, uma vez que, com o desmatamento ocorre a propagação de vetores silvestres para o meio urbano, entre tantos outros benefícios (FOLEY et al., 2005). 
Não há como atribuir a conservação do Cerrado a determinadas espécies. Algumas têm maior valor econômico diante da visão populacional. Para um estudo eficaz de uma espécie-alvo e sua conservação é necessário que se leve em conta as espécies em entorno e o ambiente que está inserido. Esta área do conhecimento é conhecida como "medicina da conservação" (SILVEIRA; D'ELIA, 2014). Entretanto, o desconhecimento acerca da biodiversidade brasileira é grande. Se tomarmos como métrica de avaliação de biodiversidade botânica os índices de suficiência amostral (quantidade de material coletado por $\mathrm{km}^{2}$, em uma determinada região), o Brasil apresenta índice de 0,7 coleta/ $/ \mathrm{km}^{2}$, inferior ao mínimo de 1,0 coleta/ $/ \mathrm{km}^{2}$, segundo o índice de Campbell e de 3,0 coletas $/ \mathrm{km}^{2}$, segundo o índice de Shepherd (SIMÕES et al., 2017). Há a necessidade de identificar e caracterizar a biodiversidade botânica (SIMÕES et al., 2000).

Desse modo, ressalta-se que o uso de espécies vegetais do Cerrado pela população local pode estimular a conservação de recursos naturais do Bioma. Isso ocorre porque a partir do momento em que os recursos naturais são utilizados diretamente, como por exemplo através do uso medicinal das espécies, ou pela comercialização, eles passam a ser protegidos pela população local (LIMA et al., 2013).

\section{Conclusões}

Notou-se que a maioria dos entrevistados utilizam plantas medicinais $(92,5 \%)$ para cura de enfermidades, as quais são indicadas principalmente pela família ou amigos. As plantas mais citadas foram barbatimão (Stryphnodendron adstringens), arnica (Lychnophora spp.) e assa peixe branco (Vernonanthura polyanthes). As indicações terapêuticas mais citadas são tratamento de doenças estomacais, gastrite e gripe. A parte da planta mais utilizada pelos entrevistados foram as folhas, seguida pela casca, raiz, entrecasca, fruto e semente.

O atual trabalho pode ser um guia para futuros estudos farmacológicos de espécies vegetais, pois indica que certas espécies têm um potencial promissor para aliviar ou curar os sintomas das doenças citadas. A aceitação de um uso terapêutico específico por uma população, implica uma maior confiança na eficácia da planta porque é mais provavelmente utilizado ou conhecido por muitos informantes. Além disso, a disseminação desses usos dentro de uma comunidade não parece ser recente, indicando que a espécie tem sido utilizada para certos sintomas ou doenças por diferentes gerações. As contraindicações citadas pelos entrevistados demonstram a necessidade de um diálogo efetivo entre profissionais especializados em saúde e comunidades locais para esclarecer o uso apropriado e os riscos de plantas medicinais.

Quanto a percepção ambiental sobre o Cerrado os entrevistados souberam definir as características gerais do bioma e reconheceram sua diminuição devido ao desmatamento, extrativismo predatório, avanço da agropecuária, clima e queimadas. Tornou-se evidente a necessidade da 
criação de projetos sociais e estudantis sobre difusão e aprendizado do conhecimento sobre conservação, manejo e cultivo das espécies locais. Este processo pode ser lento e rigoroso e necessita do envolvimento populacional e diversos profissionais como biólogos, ecólogos, zootecnistas, engenheiros ambientais e outros. Assim, é premente a conservação do Cerrado devido sua importância econômica, social e cultural. Não só para promoção do bem-estar humano, mas para preservação de fauna, fitossanitário, prevenção e cura de doenças e manutenção da prática popular de plantas medicinais pela população.

\section{Agradecimentos}

Agradecemos aos moradores da Cidade de Goiás e região pela valiosa colaboração durante as entrevistas, ao Programa de Pós-Graduação em Recursos Naturais do Cerrado e à Universidade Estadual de Goiás.

PPB, DGB, FBF e PLDA agradecem à Coordenação de Aperfeiçoamento de Pessoal de Nível Superior (CAPES) e Fundação de Amparo à Pesquisa do Estado de Goiás (FAPEG) pelas bolsas concedidas.

\section{Referências}

ALMEIDA, C.F.C.B.R.; ALBUQUERQUE, U.P. Uso e conservação de plantas e animais medicinais no Estado de Pernambuco (Nordeste do Brasil): um estudo de caso. Interciencia. v.27, n.6, 2002.

ARGENTA, S. C. et al. Plantas medicinais: cultura popular versus ciência. Vivências: Revista Eletrônica de Extensão da URI. v.7, n.1, 2011.

BADKE, M. R. et al. Medicinal plants: the knowledge sustained by daily life practice. Escola Anna Nery Revista de Enfermagem. v. 15, n. 1, 2011.

BATTISTI, C. et al. Plantas medicinais utilizadas no município de Palmeira das Missoes, RS, Brasil. Revista Brasileira de Biociencias. v.11, 2013.

BRASIL. Biodiversidade Brasileira. Ministério do Meio Ambiente. Secretaria de Biodiversidade e Florestas. Brasília: 2002. Disponível em: $<$ https://www.mma.gov.br/biomas/cerrado.html> Acesso em: 07 de outubro de 2018.

BRASIL. Política Nacional de Plantas Medicinais e Fitoterápicos. Ministério da Saúde. Secretaria de Ciência, Tecnologia e Insumos Estratégicos. Brasília: $2006 . \quad$ Disponível em: $<$ https://bvsms.saude.gov.br/bvs/publicacoes/politica nacional fitoterapicos.pdf >Acesso em: 23 de setembro de 2018.

BRASIL. Formulário de Fitoterápicos da Farmacopeia Brasileira. $1^{\underline{a}}$ ed. Agência Nacional de Vigilância Sanitária. Brasília: 2011. Disponível em: < http://portal.anvisa.gov.br/documents/33832/259456/Suplemento+FFFB.pdf/478 d1f83-7a0d-48aa-9815-37dbc6b29f9a $>$. Acesso em: 23 de setembro de 2018. 
BRASIL. Farmacopeia Brasileira: Volume 1. 5 ed. Agência Nacional de Vigilância Sanitária: Brasília, DF (Brazil), 2010. Disponível em: $<$ http://portal.anvisa.gov.br/farmacopeias-virtuais $>$. Acesso em: 23 de setembro de 2018.

BRASIL. Portaria no 2.761, de 19 de novembro. Ministério da Saúde. 2013. Disponível em: $<$ http://bvsms.saude.gov.br/bvs/saudelegis/gm/2013/prt2761 1911 2013.html >. Acesso em: 24 de setembro de 2018.

BRASIL. Memento de Fitoterápicos da Farmacopeia Brasileira. 1a ed. Agência Nacional de Vigilância Sanitária. Brasília: 2016. Disponível em: < http://portal.anvisa.gov.br/documents/33832/2909630/Memento+Fitoterapico/a8 0ec477-bb36-4ae0-b1d2-e2461217e06b> . Acesso em: 23 de setembro de 2018.

BRASIL. Formulário de Fitoterápicos Farmacopeia Brasileira - 1a edição Primeiro Suplemento. Agência Nacional de Vigilância Sanitária. Brasília: 2018a. Disponível em: $<$ portal.anvisa.gov.br/documents/33832/259456/Suplemento+FFFB.pdf/478d1f8 3-7a0d-48aa-9815-37dbc6b29f9a >. Acesso em: 24 de setembro de 2018.

BRASIL. Fauna e Flora. Ministério do Meio Ambiente. Brasília: 2018b. Disponível em: <http://www.mma.gov.br/biomas/cerrado/fauna-e-flora.html>. Acesso em: 25 de setembro de 2018.

BRASIL. Estratégia Nacional sobre Espécies Exóticas Invasoras. Comissão Nacional de Biodiversidade. Brasília: 2018c. Disponível em: $<$ https://www.mma.gov.br/biodiversidade/comissao-nacional-debiodiversidade/resolucoes > Acesso em: 25 de setembro de 2018.

BRASIL. Plano de Implementação da Estratégia Nacional para Espécies Exóticas Invasoras. Ministério do Meio Ambiente/Secretaria de Biodiversidade. Brasília: 2018d. Disponível em: < https://ambientedomeio.com/2018/08/23/plano-de-implementacao-daestrategia-nacional-para-especies-exoticas-invasoras/> Acesso em: 25 de setembro 2018.

CEOLIN, T. et al. Plantas medicinais: transmissão do conhecimento nas famílias de agricultores de base ecológica no Sul do RS. Rev Esc Enferm USP. v. 45, n.1, 2011.

CONCEIÇÃO, G. M. et al. Plantas do cerrado: comercialização, uso e indicação terapêutica fornecida pelos raizeiros e vendedores, Teresina, Piauí. Scientia Plena. v.7, n.12, 2011.

DELGADO, F. A. Goiás: A invenção da cidade "patrimônio da humanidade". Horizontes antropológicos. n.23, 2005.

DE TOLEDO, C. E. M. et al. Antimicrobial and cytotoxic activities of medicinal plants of the Brazilian cerrado, using Brazilian cachaça as extractor liquid. Journal of Ethnopharmacology. v.133, n.2, 2011. 
DIAS, J. E.; LAUREANO, L. C. Farmacopéia popular do Cerrado. $1^{\underline{a}}$ ed. Articulação Pacari: Goiás, 2010.

DOS SANTOS, M. R. A.; DE LIMA, M. R.; FERREIRA, M. D. G. R. Use of medicinal plants by the population of Ariquemes, in Rondonia State, Brazil. Horticultura Brasileira. v.26, n.2, 2008.

FOLEY, J. A. et al. Global consequences of land use. Science. v.309, 2005.

IBGE. Instituto Brasileiro de Geografia e Estatística. 2018. Disponível em: $<$ https://cidades.ibge.gov.br/brasil/go/goias/panorama>. Acesso em: $24 \mathrm{de}$ setembro de 2018.

KLINK, C. A.; MACHADO, R. B. Conservation of the brazilian cerrado. Conservation Biology. v.19, n.3, 2005.

LIMA, I. L .P.; SCARIOT, A.; GIROLDO, A. B. Sustainable harvest of mangaba (Hancornia speciosa) fruits in northern Minas Gerais, Brazil. Economic Botany. v.67, n.3, 2013.

MACÊDO, D. G. et al. Versatility and consensus of the use of medicinal plants in an area of cerrado in the Chapada do Araripe, Barbalha - CE. Brazil. Journal of Medicinal Plants Research. v.10, n.31, 2016.

MYERS, N. et al. Biodiversity hotspots for conservation priorities. Nature. v. 403, 2000.

PEREIRA, Z. V. et al. Usos múltiplos de espécies nativas do bioma Cerrado no Assentamento Lagoa Grande, Dourados, Mato Grosso do Sul. Revista Brasileira de Agroecologia. v. 7, n. 2, 2012.

PINTO, E. P. P.; AMOROZO, M. C. M.; FURLAN, A. Conhecimento popular sobre plantas medicinais em comunidades rurais de mata atlântica - Itacaré, BA, Brasil. Acta bot. Bras. v.20, n.4, 2006.

PIRES, M. O.; SANTOS, I. M. (Org.). Construindo o Cerrado sustentável: experiências e contribuições das ONG's. Gráfica Nacional: Goiás, 2000. 147p.

RAMAKRISHNAN, R. et al. In Vitro Propagation and Conservation of Useful Endangered Medicinal Plants with Anticancer Activity. J Mol Biol Biotech. v.2, n.3:8, 2017.

RODRIGUES, V. E. G.; CARVALHO, D. A. de. Levantamento etnobotânico de plantas medicinais no domínio do Cerrado na região do Alto Rio Grande Minas Gerais. Ciênc. agrotec., Lavras. v.25, n.1, 2001.

ROZA DOS SANTOS, J. G.; VESPUCCI, A. G.; BAYER, M. Qualificação dos dados hidrológicos disponíveis na base HIDROWEB/ANA: estações fluviométricas do estado de Goiás. Atelie Geogr. v.10, n.3, 2017.

SANTOS, M. M.; NUNES, M. G. S.; MARTINS, R. D. Uso empírico de plantas medicinais para tratamento de diabetes. Rev. Bras. PI. Med. v.14, n.2, 2012.

SILVA, S. D.; SÁ, D. M. de; SÁ, M. R. Vastos sertões: história e natureza na ciência e na literatura. 1 ed. Mauad X: Rio de Janeiro, 2015. 
SILVA, S. D. No oeste, a terra e o céu: a expansão da fronteira agrícola no Brasil central. 1 ed. Mauad X: Rio de Janeiro, 2017.

SILVEIRA, J. A. G.; D'ELIA, M. L. Medicina da Conservação: a ciência da saúde do ecossistema. In: JUNIOR, A. P. M. et al. Ecoas: Epidemiologia e conservação de animais silvestres. Cadernos Técnicos de Veterinária e Zootecnia, n.72, 2014.

SIMÕES, C. M. O. et al. (Org.). Farmacognosia: da Planta ao Medicamento. 2 Ed. Editora UFRGS: Porto Alegre, 2011.

SIMÕES, C. M. O. et al. (Org.). Farmacognosia: do Produto Natural ao Medicamento. 1 ed. Editora UFRGS:Porto Alegre, 2017.

SOUZA, C. D.; FELFILI, J. M. Uso de plantas medicinais na região de Alto Paraíso de Goiás, GO, Brasil. Acta Bot. Bras. v.20, n.1, 2006.

TEIXEIRA, A. B.; DUTRA E SILVA, S.; DARC BERNARDES, G. Patrimônio cultural e turismo sustentável: Expectativas e percepções na gestão turística da cidade de Goiás. Tourism \& Management Studies. v.1, 2012.

TOMAZZONI, M. I.; NEGRELLE, R. R. B.; CENTA, M. L. Fitoterapia popular: a busca instrumental enquanto prática terapêuta. Texto contexto enfermagem. v.15, n.1, 2006.

UNNIKRISHNAN, P. M.; FADEEVA, Z. Innovation in Local and Global Learning Systems for Sustainability: Traditional Knowledge and Biodiversity - Learning Contributions of the Regional Centres of Expertise on Education for Sustainable Development. UNU-IAS, Yokohama, Japan, 2013.

UNNIKRISHNAN, P. M.; SUNEETHA, M. S. Biodiversity, Traditional Knowledge and Community Health. 1 ed. Strengthening Linkages: Singapore, 2012.

WILLIAMS, V.L.; BALKWILL, K.; WITKOWSKI, E.T.F. Unraveling the commercial market for medicinal plant parts on the Witwatersrand, South Africa. Economic Botany. v.54, n.3, 2000. 